\title{
A GIS-based Spatial Decision Support Tool Based on Ex- tended Belief Rule-Based Inference Methodology
}

\author{
Alberto Calzada ${ }^{1}$, Jun Liu ${ }^{1}$, Hui Wang ${ }^{1}$, Anil Kashyap ${ }^{2}$ \\ ${ }^{1}$ School of Computing and Mathematics, University of Ulster, UK \\ ${ }^{2}$ School of the Built Environment, University of Ulster, UK \\ Calzada-A@email.ulster.ac.uk, \{j.liu, h.wang, a.kashyap\}@ulster.ac.uk
}

\begin{abstract}
Nowadays, most Spatial Decision Support Systems (SDSSs) are designed to solve a specific problem in a given region. This fact makes rather difficult or even impossible to develop comparative analyses and studies among different solutions. This research presents a generic rule-based spatial decision support software tool, able to approach most spatial decision problems within a single framework. To achieve this, the rule-based RIMER+ decision model was embedded in a Geographic Information System (GIS) environment. Such system was named Spatial RIMER+, and is able to consider expert knowledge, data uncertainty and both spatial and nonspatial information during the decision making process.
\end{abstract}

Keywords: Spatial Decision Support System, RIMER+, GIS, Extended Belief-Rule Base

\section{Introduction}

Most real-case complex decision problems can be considered as spatial decision problems, since they are usually located in a certain area and have a spatial component. In such situations, both data location and spatial relationships usually make a prominent impact during the decision making process. For example, decisions related with urban regeneration, environmental risk assessment or policy making contain a strong spatial component that should be analyzed and included as a new dimension into the decision making process (Sugumaran and DeGroote, 2010). Therefore, spatial decision problems should be geographically referenced and represented in a computer by using Geographic Information Systems (GIS). In some cases, decision makers rely just on the GIS powerful storage and visualization capabilities to obtain support for their decisions (Thomas and Humenik, 2009), leaving the analysis and interpretation tasks to the decision maker.

However, such complex spatial decision problems require considering a large set of parameters, policies, expert and stakeholder opinions and alternatives in a dynamic environment. As the complexity of the problem increases, it becomes difficult or even impossible to plot all the digital information to be analyzed in a GIS environment (Sugumaran and DeGroote, 2010; Malczewski,
2006). Besides, other aspects like data uncertainty, heterogeneity, completeness or similarity must be taken into account in order to increase the quality and precision of the decision output. Although Decision Support Systems (DSSs) are the computational response to such complex decision problems, usually they are not designed to take into account the spatial dimension. In this regard, Spatial Decision Support Systems (SDSSs) have been increasingly used in the last 15 years or so (Sugumaran and DeGroote, 2010; Malczewski, 2006).

In this research, the RIMER+ decision model (Liu et al, 2012; Liu et al, 2013) has been considered as the most suitable approach that meets most complex decision making challenges. This is so because its knowledge base consists of sets of extended belief rules, named Extended Belief Rule Bases (E-BRBs), which are able to capture non-linear relationships among data as well as a heterogeneous information, incompleteness and uncertainty (Liu et al, 2012; Liu et al, 2013). This last aspect is especially relevant because recent studies have shown that most SDSSs are based on deterministic approaches (Malczewski, 2006), leaving aside the uncertainty aspect, which is intrinsic to most real-world spatial decision problems.

Some fuzzy modeling techniques have been applied to integrate uncertainty as an integral component to solve spatial decision problems in the last years, like the works of (Chang et al., 2008; Li et al., 2005; Girvetz and Shilling, 2003). However, the vast majority of them are problem-specific applications, difficult to export to different problem domains to perform comparative studies (for example, in terms of accuracy). Maybe the only system based on a generic approach is the one presented by (Boroushaki and Malczewski, 2008). Their method is based on a combination of the Analytic Hierarchy Process (AHP) and the Ordered Weighted Average (OWA), but its user interface is not intuitive. For example, the hierarchy of attributes and goals is not depicted, so for large spatial decision problems (which are the majority in realcase scenarios) it would be difficult for the end-user to understand the broad picture of the problem. Moreover, their system has been built on top of GIS proprietary software (ArcGIS) and requires experts and decision makers to manually input most information for each attribute and goal, which can be exhausting and imprecise. 
The generic SDSS proposed in this paper was built on top of open-source GIS, which makes it potentially freely available, and thanks to the straightforward rule generation scheme of RIMER+, the system inputs most information automatically by learning from the GIS data available when building the rules in the attributes hierarchy.

The RIMER+ method has been recently upgraded with a Dynamic Rule Activation (DRA) process, which is able to detect and avoid common issues in spatial decision problems, such as data incompleteness and inconsistency (Calzada et al, 2013a; Calzada et al, 2013b). After DRA evaluates the rule activation process, the Evidential Reasoning (ER) algorithm, included in the RIMER+ method, infers a decision outcome.

This paper presents a Spatial Decision Support Tool, based on the RIMER+ methodology to deal with complex decision support issues and allocated in a GIS environment in order to integrate the spatial component of such complex spatial decision making. The software tool, named Spatial RIMER+, aims to facilitate the spatial decision problems definition, implementation and solving in an integrated environment. Such tool needs to establish a full communication channel between the GIS and DSS systems. To achieve so, the RIMER+ methodology was embedded into the GIS environment. In this way, both spatial and non-spatial dimensions of the problem can be handled at once with a single software tool.

In general terms, embedding RIMER+ in a GIS environment allows two levels of integration of the spatial dimension of the problem: (i) by taking advantage of the GIS capabilities in terms of data storage, management and most specially visualization, in order to provide ways to learn from the non-spatial information included in the GIS system databases and "manually" analyze spatial patterns in the decision outcomes and (ii) by actually considering spatial information as an integral part of the decision process, as some of the most relevant spatial-based regression models do (Charlton et al, 2009). This paper presents the Spatial RIMER+ software tool; therefore the main focus is on the first level of integration. For more details about the second level of integration, see (Calzada et al., 2013c).

The remaining of this paper is organized as follows: Section 2 briefly outlines the RIMER+ approach; Section 3 details the system architecture and describes how the integration of RIMER+ into the GIS is performed; Section 4 explains how a spatial decision problem can be defined and approached with the Spatial RIMER+ software tool and Section 5 presents a case study showing the main functionality of the system prototype. Finally, Section 6 concludes the paper.

\section{Outlining RIMER Approach}

RIMER (Yang et al., 2006) presents a belief rule representation scheme to extend traditional IF-THEN rules using a belief structure. The belief rule base (BRB) intro- duced in RIMER approach is designed with belief degrees embedded in the consequent terms of the rules. Recently, this scheme has been further upgraded as a new belief rule base with belief degrees embedded in the entire consequent terms as well as in the entire antecedent terms of each rule (Liu et al., 2012; 2013). Such rules have been named Extended Belief Rules and are grouped forming Extended Belief Rule-Bases (E-BRBs). The decision model considering these E-BRBs was named RIMER+.

Both BRBs and E-BRBs (Liu et al., 2012; 2013) are designed to meet the need of handling uncertain, non-linear and heterogeneous information in human decision making. However, E-BRBs have a higher representation power, since they incorporate belief degrees embedded in all the antecedents and consequent terms of a rule, easing the rule generation and direct input transformation tasks. Thanks to these belief degree distributions, the direct input transformation is achieved by using the linguistic terms defined for each antecedent or consequent attribute, which can be defined as one of the following cases: (1) matching function methods using fuzzy membership functions; (2) rule-based or utility transformation methods; or (3) subjective valuation methods, for attributes of qualitative nature. By using one of these 3 options, every input can be directly transformed and normalized as a belief distribution form, with no loss of information during the process. Note that this direct input transformation is able to easily standardize information from different sources and nature in a common framework. In this regard, an E-BRB can be generated by using information from different sources, like available databases, expert knowledge or vague opinions and judgments. This direct input transformation will also be essential when using the E-BRB model in a GIS environment, since the spatial and non-spatial information included in the GIS tool can be used to generate new extended belief rules. Moreover, all these sources of information can be integrated together considering other essential factors in real-world problems, like data incompleteness, vagueness and uncertainty. For example, consider the following extended belief rule:

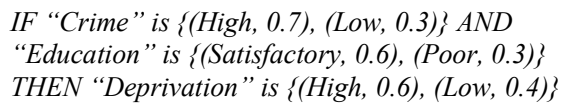

The "Education" antecedent has a belief degree of $60 \%$ for the "Satisfactory" term and $30 \%$ for the "Poor" one, leaving undefined the remaining $10 \%$. This undefined part represents the incompleteness degree of the antecedent. In this context, E-BRBs are normally used to integrate in the decision process some key elements of realworld decision problems, like vagueness (with linguistic terms), uncertainty (with beliefs), information incompleteness (partially known beliefs in antecedents and/or consequents) and nonlinear relationships between indicators (with IF-THEN rules; between its antecedents and the consequent). They also provide a flexible way to incorporate hybrid input information (both quantitative and qualitative) as well as an efficient rule generation scheme (Liu 
et al., 2012; 2013). All these aspects of E-BRBs become essential when trying to deliver useful support in complex real-world problems.

In this context, RIMER+ can be defined as a decision model that selects relevant rules from the E-BRBs and aggregates it using the Evidential Reasoning (ER) inference algorithm to provide a decision output (Liu et al., 2012; 2013). To enhance the rule selection process, RIMER+ has been recently upgraded with a Dynamic Rule Activation (DRA) pre-processing step that helps avoiding situations of inconsistency and incompleteness in the rule-base (Calzada et al., 2013a; 2013b). DRA is an adaptive algorithm that evaluates and adjusts the activated rules to be inferred by ER afterwards. The studies presented in this work take advantage of this method to demonstrate some of the key points of spatial decision problems.

Because of all the positive features included in E-BRBs, the ER algorithm as well as in the DRA approach, the RIMER+ framework is considered as a solid methodology to approach complex decision problems (Lui et al, 2013; Liu et al, 2012). However, as real-case scenarios are usually located in a particular area of study, the spatial component of the problem needs to be incorporated in the decision support analysis. This fact adds a new dimension to the problem, and therefore the spatial side of the problem needs to be considered in the decision making process. Next Section illustrates how this integration was designed in order to implement the Spatial RIMER+ software prototype.

\section{Spatial RIMER+}

Spatial RIMER+ is a GIS-based spatial decision support system that makes use of the RIMER+ methodology by embedding it in a GIS environment. In order to deliver decision support in complex real environments, the spatial dimension of the problem becomes an essential component to be considered. In the Spatial RIMER+ method, two levels of the spatial integration are considered (Calzada et al., 2013c): (i) taking advantage of the storage and visualization tools provided in the GIS software, embedding the decision process within it and (ii) by actually incorporating spatial data in the decision process to automatically analyze it. Since this paper focuses on presenting a spatial decision support system prototype, the first integration level will be exhaustively described. For more technical information about how the second level of spatial analysis integration is achieved within the framework of this research, see (Calzada et al., 2013c).

To achieve the first level of integration, software tools need to be developed in order to establish a full communication protocol between the GIS and DSS software tools. Following subsections briefly illustrate the software architecture, data structures and communication protocols underneath the Spatial RIMER+ software tool.

\subsection{GIS-based Decision Support System Architec-} ture

Two different, specialized computer solutions are needed when trying to implement a SDSS: (i) a GIS system that provides spatial database management, spatial information and visual support to analyze spatial patterns in data and (ii) a DSS that involves other important analyses and inference methods taking into account data similarity, uncertainty and incomplete and inconsistent situations. In the Spatial RIMER+ system, the latter requirement is filled by using the RIMER+ decision model.

The open-source system provided by the gvSIG project (gvSIG Association, 2010; Anguix et al., 2008) was utilized to bring the GIS capabilities into the Spatial RIMER+ system. gvSIG is freely available in the website of the gvSIG project. Like the RIMER Tool (Calzada et al., 2011), gvSIG is developed in the Java language. Therefore, the system was designed under the objectoriented programming paradigm, so abstract concepts can be defined and grouped into packages. This fact considerably eases the design and implementation stages of the Spatial RIMER+ software, providing a large range of possibilities to integrate the two systems within a common framework.

The general structure of the RIMER Tool packages follows a Model-View-Controller design (Calzada et al., 2011). Although it is not required to modify this structure when upgrading the system to be embedded into the GIS software, some additional packages need to be created, in order to establish communication protocols between the two software tools. Figure 1 below illustrates the general architecture of the system:

For further details about the gvSIG packages illustrated in Figure 1, see (gvSIG Association, 2010). Note that the RIMER+ software prototype is an extended version of the system presented in (Calzada et al., 2011). Next subsection describes how the two different software systems work together to deliver decision support.

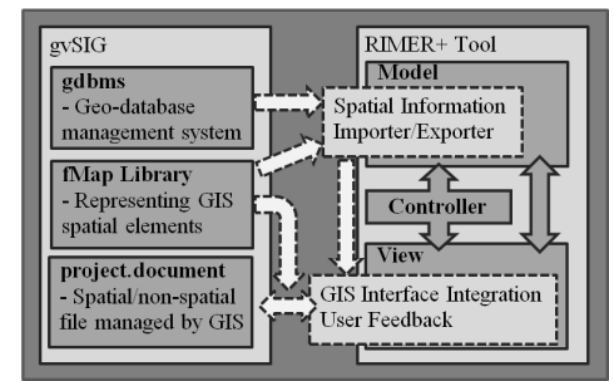

Figure 1. Overall Software Architecture of Spatial RIMER+. Light components are the packages (represented in dashed rectangles) and methods (dashed arrows) that allow the communication between the two systems

\subsection{Spatial RIMER+ Subsystems Communication}

In order to provide a full communication between the two software systems in a common framework, this research proposes an embedded approach, including the DSS as an 
extension of the GIS software. The first step to combine such different systems is collecting and formatting spatial data from the GIS to be used in the decision making process. E-BRBs provide a solid starting point to achieve this task. As mentioned in previous sections, E-BRBs input transformation is a straightforward procedure without any loss of information, so all the information from the original spatial data can be transmitted to each extended belief rule. Therefore, each piece of information included in the GIS spatial database can be easily transformed as an extended belief rule. Note that the scenario of a spatial problem can include data not only from the GIS source, but also be further specified. This task can be easily done in the RIMER+ decision model by adding more extended belief rules automatically from different data sources or manually, using expert knowledge.

Once the E-BRBs of the problem are generated, extended belief rules can be activated for a given set of inputs (spatially located or not) within the RIMER+ decision framework, in order to compute a decision outcome. Then, this output can be returned to the GIS system and directly mapped using the GIS powerful visualization methods. Several decision outputs can be plotted in the same way in order to visually analyze patterns, clusters and other spatial phenomena that could be difficult to obtain otherwise. Figure 2 summarizes the Spatial RIMER+ system architecture, and how the information between both GIS and DSS flows within a common environment.

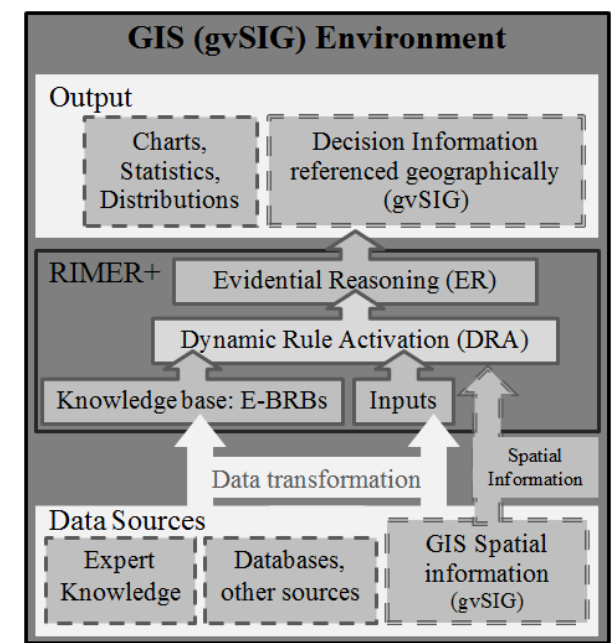

Figure 2. Integration of RIMER+ into a GIS environment

Next Section presents a step-by-step case study showing how this flow of information is performed when approaching a spatial decision problem using the Spatial RIMER+ Tool.

\section{Spatial Decision Making in Spatial RIMER+ Tool}

This Section illustrates how common different tasks associated with spatial decision problems can be performed using the Spatial RIMER+ software prototype. These tasks are grouped in two main processes: (i) problem definition and (ii) solving process.

\subsection{Problem Definition}

The Spatial RIMER+ system provides the flexibility needed to define most spatial decision problems. This functionality ranges from several choices when defining attributes to hierarchical decision structure generation and many possibilities to import data from different sources. To provide this functionality in the user interface, the RIMER+ Tool includes two main windows: the attribute window and the main window, used for the hierarchical structure management. Figure 3 below shows a caption of both windows:

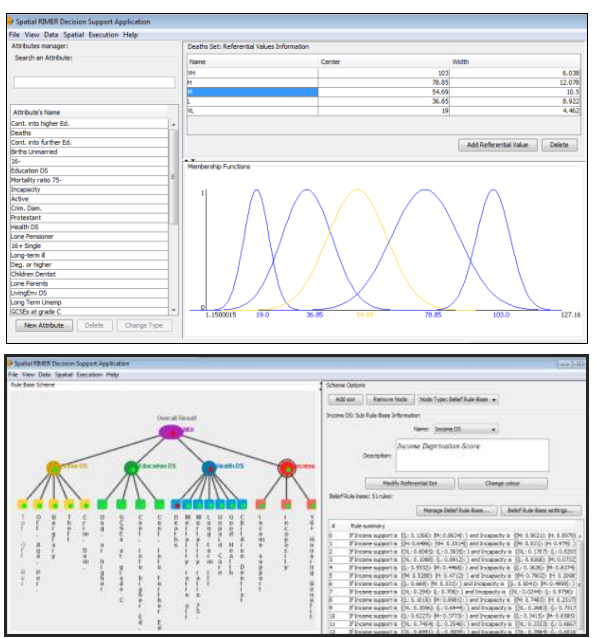

Figure 3. Attributes management window (top) and main window (bottom)

\section{Attribute Definition}

The attributes management window manages every aspect regarding the attributes and their referential values and membership functions. Spatial RIMER+ allows defining attributes using several types of functions in order to represent attributes from different nature. Figure 4 summarizes the options included:

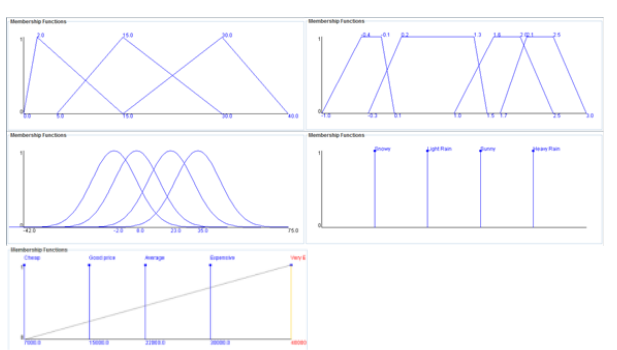

Figure 4. Possible membership functions for attributes in Spatial RIMER+. From top-left: triangle and trapezoid fuzzy membership functions, Gaussian fuzzy membership function, qualitative values and utility function. 
Although these functions usually need to be manually defined, the Spatial RIMER+ software is able to generate them automatically when importing data from the GIS system.

\section{Problem Structure}

In the main window, it is possible to define a hierarchical structure of the decision problem by adding/removing nodes (See Figure 3, bottom caption). Each one of these nodes will be associated with one of the attributes previously generated. Leaf nodes in the hierarchy will be inputs for the system, while intermediate and root nodes will represent the relationship between their sons by means of an E-BRB or simply defining a mathematical function. See (Calzada et al., 2011) for more information about the attribute definition and problem structure processes.

\section{Data Import}

Once attributes and structure of the decision problem are defined, the last step to complete the decision problem definition is to generate extended belief rules. Taking advantage of the straightforward rule generation scheme provided by the E-BRBs, the Spatial RIMER+ software tool provides several ways of adding information to the knowledge bases included in the hierarchical structure: (i) manual rule creation, based on expert knowledge, (ii) automatic generation based on other data sources, like Excel files and (iii) automatic generation from the geographic database of the GIS system.

In this way, all the relevant information from different sources and nature is homogenized within a common framework. This fact greatly enhances the overall performance of the system, since most of the aspects of spatial decision problems can be considered using one methodology within a single software tool. It is important to note that usually, the most information is included in the EBRBs, the better RIMER+ can learn from data and therefore the most accurate the decision outcome will be. In this regard, the Spatial RIMER+ tool provides the functionality needed to add as much relevant information as needed in order to approach complex spatial decision problems.

\subsection{Solving Process}

Having the spatial decision problem defined using attributes organized in a hierarchy and the knowledge represented into the E-BRBs and mathematical functions, the next step is to produce decision results using the Spatial RIMER+ system.

\section{Test Definition and Execution}

Taking advantage of the uncertainty management provided in the RIMER+ method, several types of inputs with different degrees of uncertainty can be assigned in the Spatial RIMER+ system: (i) numerical values, (ii) interval values, (iii) a linguistic term included in the attribute definition, (iv) a membership function defining a new linguistic term or (v) a probabilistic belief degree distribution.

The system can be tested in several ways: (i) manually include inputs to get a single result, (ii) test the accuracy of the E-BRBs using the cross-validation method, (iii) using some knowledge included in the E-BRBs for testing and the remaining for training or (iv) introduce a set of inputs from an external source to produce a series of decision results.

For the first test case, a single output for the set of inputs manually defined will be provided. In the remaining cases, a set of decision outputs will be retrieved. In the case that the inputs and the E-BRB have been generated from the GIS software and have spatial capabilities, the outputs will be mapped in the GIS system. This functionality will have a great use to further analyze geographic patterns and ease the complex decision making process for the end user.

\section{Output Retrieval and Analysis}

As aforementioned, in the case that spatial information is used; the decision outputs will be plotted in the GIS environment. Otherwise, the outputs will be represented within the RIMER+ Tool or exported to Excel CSV format. This research focuses on the spatial features of Spatial RIMER+; therefore only the spatial case will be considered here.

Once the RIMER+ outputs are exported in GIS format, it is possible to take advantage of the GIS capabilities to present them in a visually appealing way. Moreover, Spatial RIMER+ includes methods to visualize the GIS output in belief degree distribution form. For research purposes, it is also possible to compare the RIMER+ result(s) with the real value(s) of the variable. Figure 5 shows how these comparative analyses are represented for the case when a single output (top) and a set of outputs (bottom) are selected:

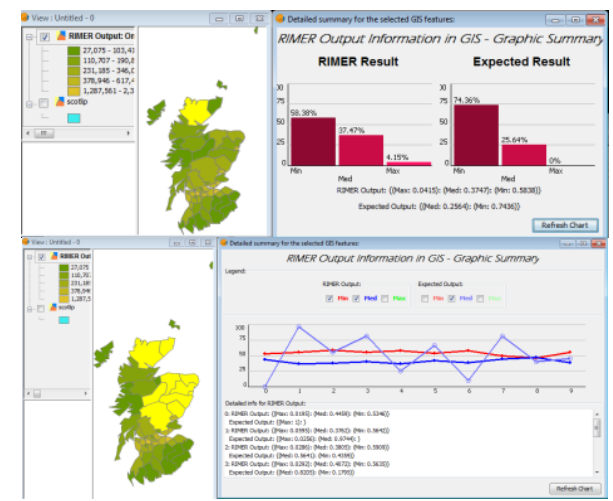

Figure 5. The RIMER+ outputs are automatically exported as a GIS layer. Map source: (GeoDa Centre, 2006) 
As illustrated in Figure 5, thanks to the GIS technology, several colors can be used to represent different values of the output variable and analyze spatial patterns. Then, the user can select one or more outputs and plot them in different charts in order to check the accuracy of the RIMER+ method by comparing it to the real value of the output variable.

\section{Case Study}

To illustrate the capabilities of the Spatial RIMER+ software tool, a case study using real-data about private transport features and traffic collisions was performed. The data was extracted from the publicly available NISRA database (NISRA, 2013). The aim of the study is to analyze whether the features of the vehicles registered in a certain area can be used to predict the number of traffic collisions occurring in that area, in order to deliver useful support for many aspects like road maintenance, investment or policy decision making, in order to prevent traffic accidents in the future.

For illustrative purposes, just five variables were selected to predict the number of traffic accidents happening in a certain area. The five variables are number of vehicles registered (1) with more than 3000 cubic centimeters of power, (2) for disabled people, (3) with a non-standard color, (4) before year 2000 and (5) recently, from 2009 onwards. While these 5 variables correspond to data from the year 2011, the dependent variable (number of traffic collisions) is from the year 2012. Therefore, the study analyses how the results forecasted in 2011 match with the real figures of 2012. In case that the accuracy of the system is good enough, similar data from the year 2013 could be used to predict traffic accidents of 2014, and so on.

To define this spatial decision problem, the GIS layer containing the information about vehicles registered and a traffic collision was opened. Then, the Spatial RIMER+ system automatically reads all the variables included in the layer and generates their utility functions. These utility functions can then be tuned or modified by the user. Finally, the values for each variable and each spatial location were automatically loaded as extended belief rules in the root of the hierarchy (See Figure 6).

Once the attributes, structure and rules are defined in the system, the last step is to perform some test to check the accuracy of the E-RBB, which will be based on the quality and quantity of information loaded from the GIS tool. In this case, a 10-fold cross-validation test was performed, and the results were exported to the GIS software. The test to predict the 582 values for the traffic collisions in each electoral ward of Northern Ireland, using the DRA enhancement for the E-BRB, lasted for 4 minutes and 18 seconds in an Intel Core i3 CPU of $2.53 \mathrm{GHz}$. The RIMER+ results were then plotted in the GIS map, with the areas were RIMER+ believes that more accidents occur in darker colors. Figure 7 illustrates the Spatial RIMER+ output.

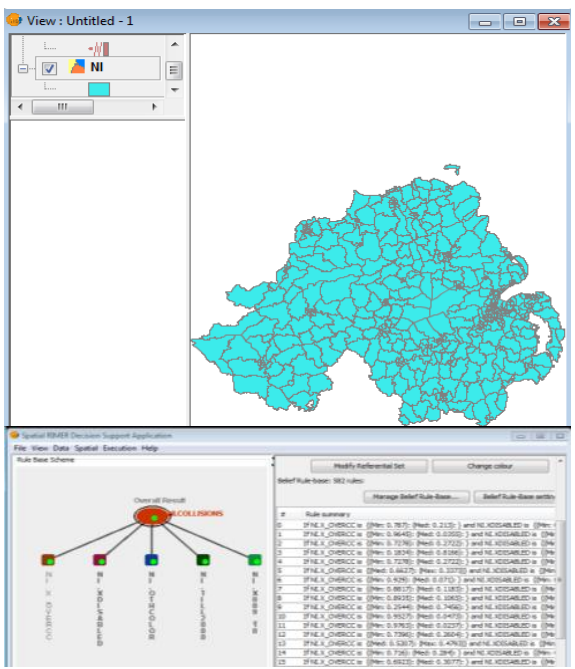

Figure 6. Caption of the Spatial RIMER+ system with the rules automatically generated for the hierarchy and included in the EBRB of the "Collisions" attribute

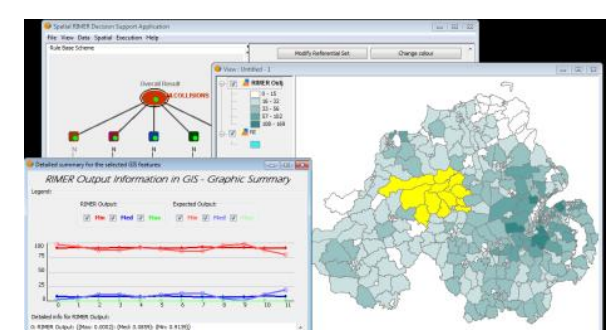

Figure 7. Spatial RIMER+ decision outputs. Zones in darker colors usually have more accidents. The areas highlighted in yellow were selected by the user, to plot them in the line chart on the left, to check the accuracy of RIMER+

In this case study, the Mean Absolute Error (MAE) of the RIMER+ method was of just 5.36. RIMER+ performance has been demonstrated in several publications (Liu et al., 2012; Calzada et al., 2012a; 2012b), and further improved by using the DRA method (Calzada et al., 2013a; 2013b). Once the decision outputs are exported to the GIS database, a further analysis can be performed for research purposes. For example, the absolute error in each area can be easily plotted, as in Figure 8:

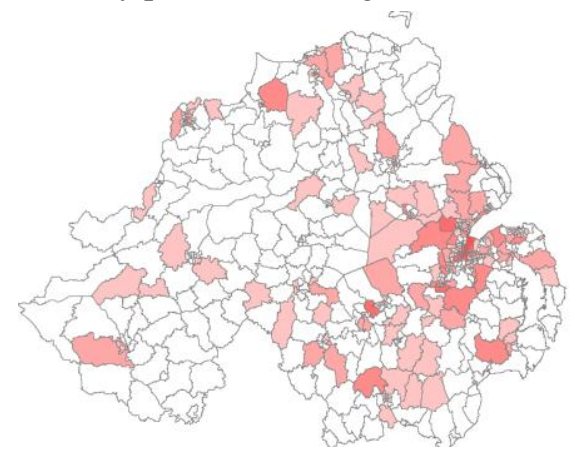

Figure 8. Absolute error of RIMER+ in each region 
In the areas colored in white, RIMER+ predicted the result accurately, while in zones colored in darker red RIMER+ had some error in the prediction. Note that the Spatial RIMER+ tool can be used not only to deliver useful decision support, but also open many lines of research in the field of spatial decision making. Analyzing the reasons why some classifiers are not able to provide more accurate predictions in certain areas is a topic to be further explored. In this regard, some research attempting to include spatial information within the decision making process is being performed (Calzada et al., 2013c).

\section{Conclusions}

This research presented Spatial RIMER+; a spatial decision support software tool, able to deliver useful decision making in spatial environments. Such spatial decision support system consists of two main components: (i) the gvSIG software system, to provide GIS capabilities in terms of data storage, management and visualization and (ii) the RIMER+ decision model that is able to consider other essential elements of spatial decision problems, like data similarity, uncertainty, incompleteness and inconsistency within a common framework. RIMER+ also eases the analysis task, by providing an inference algorithm that aggregates the most relevant information for a set of inputs in order to deliver a decision output. These decision outputs can be plotted in the GIS environment in order to analyze spatial correlation, homogeneity and other patterns in situ.

Spatial RIMER+ can be extensively used for research purposes. The software tool provides all the functionality required in order to measure, represent and interpret the system accuracy, error statistics, correlation analysis, test definition, performance and many other useful procedures that are commonly used in research in order to perform case studies and comparative analysis.

\section{References}

[1] Anguix A, Díaz L., and Carrera M.. (2008) "gvSIG: A GIS desktop solution for an open SDI." Journal of Geog. and Reg. Plan. 1.3: 041-048.

[2] Brunsdon C., Fotheringham S., and Charlton M. (1998) "Geographically weighted regression." Journal of the Royal Statistical Society: Series D (The Statistician) 47.3: 431-443.

[3] Calzada A., Liu J. and Wang H. (2013a) "A new dynamic rule activation method for extended belief rule-bases", Submitted to IEEE Transactions on Knowledge and Data Engineering under review.

[4] Calzada A., Liu J., Wang H. and Kashyap A. (2013b) "Dynamic rule activation for extended belief rule bases", Int. Conf. in Machine Learning and Cyber. (ICMLC2013), July 14-17, 2013, Tianjing, China.
[5] Calzada A., Liu J., Wang H. and Kashyap A. (2013c) “A Novel Spatial Belief Rule-Based Intelligent Decision Support System", accepted at IEEE Intl Con. on Systems, Man and Cybernetics (IEEE SMC 2013), October 13-16, 2013, Manchester, UK.

[6] Calzada A., Liu J., Wang H. and Kashyap A. (2012a), "An empirical comparative study for urban regeneration: measuring the effectiveness of DSS and GIS approaches", presented at the 19th Annual European Real Estate Society Conference (ERES 2012). Edinburgh, Scotland, June 13-16, 2012

[7] Calzada A., Liu J., Wang H., Kashyap A. (2012b), "Uncertainty and incompleteness analysis using the RIMER approach for urban regeneration processes: the case of the Greater Belfast Region", International Conference on Machine Learning and Cybernetics (ICMLC 2012), Xian, P.R. of China 15-17 July, 2012

[8] Calzada A., Liu J., Wang H., Martínez L., Kashyap A. (2011) "An intelligent decision support tool based on belief rule-based inference methodology". IEEE International Conference on Fuzzy Systems. Taipei, Taiwan. June 27-June 30, 2011

[9] Charlton M., Fotheringham S., and Brunsdon C. (2009) "Geographically Wweighted Rregression." White paper. National Centre for Geocomputation. National University of Ireland Maynooth.

[10] Chang N.B., Parvathinathan G., Breeden J.B., (2008), "Combining GIS with fuzzy multi-criteria decisionmaking for landfill siting in a fast-growing urban region", Journal of Environmental Management, 87:139153.

[11] GeoDa: Anselin L., Syabri I., and Kho Y.. (2006) "GeoDa: An introduction to spatial data analysis." Geographical Aanalysis 38.1: 5-22.

[12] Givertz E., and Shilling F, (2003), "Decision Support for road system analysis and modification on the Tahoe National Forest". Environmental Management. $32(2): 218-233$.

[13] gvSIG Association (2010) "gvSIG Desktop 1.11." User Manual.

[14] Li L., Wang J. and Wang C., (2005), “Typhoon insurance pricing with spatial decision support tools". International Journal of Geographical Information Science, 19 (3): 363-384

[15] Liu J., Martinez L., Calzada A., and Wang H. (2013), "A novel belief rule base representation, generation and its inference methodology", Knowledge-Based Systems, accepted and in press.

[16] Liu J., Martinez L., Wang H., Calzada A, and Chen S.W. (2012), "A new belief rule base representation scheme and its generation by learning from examples", Proceeding of the 10th International FLINS Conference on Uncertainty Modelling in Knowledge Engineering and Decision Making (FLINS2012), Istanbul, Turkey, 27-29th, August, 2012.

[17] Malczewski, J. (2006) "GIS- based multi-criteria decision analysis: a survey of the literature." Intl J. of Geog. Inf. Science 20.7: 703-726.

[18] NISRA (2013). Northern Ireland Statistics and Research Agency (NISRA). National Statistics, Crown 
copyright. [Available at: http://www.nisra.gov.uk/, Accessed 20th, May, 2013].

[19] Sugumaran R. and DeGroote J., "Spatial Decision Support Systems: Principles and Practices", CRC Press.

[20] Thomas C. and Humenik N. (2009) "GIS for Decision Support aAnd Public Policy Making.” Redlands, Calif.: ESRI Press.

[21] Yang J.B., Liu J., Wang H., Sii S. and Wang H.W. (2006), "A generic rule-base inference methodology using the evidential reasoning approach - RIMER", IEEE Transactions on Systems, Man and Cybernetics-Part A: Systems and Humans, 36.2: 266-285. 